\section{Striving toward malaria elimination and the necessity to be persistent in our efforts}

\section{Saurabh R. Shrivastava, Prateek S. Shrivastava, Jegadeesh Ramasamy}

Department of Community Medicine, Shri Sathya Sai Medical College and Research Institute, Kancheepuram, India

Across the world, malaria continues to remain a major cause of public health concern as even now $50 \%$ of the world's total population is at potential risk of malaria. ${ }^{1}$ Out of the 106 nations which were facing the challenge of malaria at the start of the current century, recent estimates suggest that almost 57 and 18 of them have achieved 75 and $50-75 \%$ reduction in the incidence of malaria, respectively. ${ }^{2}$ However, the African region alone accounts for $88 \%$ of the total malaria cases and $90 \%$ of the overall deaths in the year $2015 .^{3}$

Moreover, owing to the implementation of targeted approach a significant decline in both the global incidence by more than one-third, and mortality rates by three-fifth $(0.83$ million deaths in 2000 versus 0.43 million deaths in 2015) in the span of last 15 years has been accomplished. ${ }^{2,3}$ Further, population groups of under-five children, antenatal mothers and susceptible persons going to the malaria endemic regions are extremely vulnerable to the disease. ${ }^{1}$ The recent estimates reflect that a $65 \%$ decline in the malarial death rates has been observed among the under-five year children age-group on the global scale., ${ }^{1,2}$

In addition, an expenditure of millions of dollars has been saved, especially with regard to the management of the patients since $2000 .^{2}$ Also, an excess of 6 million deaths across the world and more than 650 million cases of malaria (African region alone) have been prevented in the last 15 years. ${ }^{3}$ Most of the current trends reflect that significant improvement has been achieved towards the ultimate goal of malaria elimination. ${ }^{45}$ In-fact, the international stakeholders were even successful in accomplishing the proposed malaria target under the
Millennium Development Goal - 6.,4

Although, many factors have played their part in ensuring an improvement, the predominant share of the achieved success goes to the sustained level of political commitment, technical assistance from the World Health Organization (WHO), and financial support from the earmarked international agency. ${ }^{3-5}$ However, among the implemented measures, three low-cost strategies have delivered maximum output, namely insecticide-treated mosquito nets (maximum contribution in financial savings), artemisinin-based combination therapies (highly effective treatment regimen for falciparum malaria) and indoor residual spraying. ${ }^{2,3}$ Further, a significant rise in the incidence of rapid diagnostic testing for malaria has also been reported from heterogeneous settings. ${ }^{1,4}$

However, even now, many challenges persist and there is a great need to address all of them. ${ }^{1,3,5}$ These include high caseload and death rates, localization of maximum number of cases from some of the high-burden nations, a slow rate of decline in the incidence/death rates of malaria compared to the global trends, shortcomings in the health care system, inaccessibility of the services (like mosquito nets or indoor residual spraying), emergence of drug resistance and insecticide resistance, and poor involvement of the community stakeholders..$^{1,3,5,6}$ Finally, a newer strategy has been adopted by the WHO member states to reduce the global malaria incidence and mortality by at least $90 \%$, eliminate malaria from 35 nations, and to prevent the re-emergence of the disease in malaria free nations by the year 2030.1,3

To conclude, now the goal is set, we have access to effective measures which have delivered results, but the challenge is to implement the same for a longer duration of time. Thus, the need of the hour is to have a strong leadership at different levels, constant motivation of the health workers, strategic involvement of the community, and up-scaling of the financial support.

\section{References}

1. WHO. World malaria report 2015. Geneva,
Correspondence: Saurabh RamBihariLal Shrivastava, Department of Community Medicine, Shri Sathya Sai Medical College and Research Institute, Thiruporur-Guduvancherry main road, 603108 Kancheepuram, India.

Tel./Fax: +91.988.422.7224.

E-mail: drshrishri2008@gmail.com

Contributions: SRS, conception and design, drafting of the article, review of literature, guarantor; PRS, drafting the article, review of the literature, critically revising for important intellectual content; JR, general supervision of the research, overall guidance in writing the manuscript.

Conflict of interest: the authors declare no potential conflict of interest.

Key words: Malaria; Insecticide-treated nets; World Health Organization.

Received for publication: 18 January 2016. Accepted for publication: 6 February 2016.

This work is licensed under a Creative Commons Attribution 4.0 License (by-nc 4.0).

○Copyright S.R. Shrivastava et al., 2016

Licensee PAGEPress, Italy

Healthcare in Low-resource Settings 2016; $4: 5755$ doi:10.4081/hls.2016.5755

Switzerland: WHO; 2015.

2. WHO. Malaria. Available from: http://who.int/mediacentre/factsheets/fs09 4/en/

3. WHO. Achieving the malaria MDG target: reversing the incidence of malaria 20002015. Geneva, Switzerland: WHO; 2015.

4. Chanda E, Ameneshewa B, Angula HA, et al. Strengthening tactical planning and operational frameworks for vector control: the roadmap for malaria elimination in Namibia. Malaria J 2015;14:302.

5. Hsiang MS, Gosling RD. Striding toward malaria elimination in China. Am J Trop Med Hyg 2015;93:203-4.

6. Whittaker M, Smith C. Reimagining malaria: five reasons to strengthen community engagement in the lead up to malaria elimination. Malaria $\mathrm{J}$ 2015;14:410. 